\title{
Odontogenic origin of descending necrotizing mediastinitis - controversies, multidisciplinary approach and personal experience
}

Agnieszka Kozak-Rusinek ${ }^{1}$, Katarzyna Kozak², Michał Rusinek ${ }^{3}$, Marcin Kozakiewicz', Jozef Kozak ${ }^{4}$

\author{
${ }^{1}$ Department of Maxillofacial Surgery, Medical University of Lodz, Lodz, Poland \\ 2Department of Nephrology, Hypertension and Family Medicine, Medical University of \\ Lodz, Lodz, Poland \\ ${ }^{3}$ Department of Urology, Medical University of Lodz, Lodz, Poland \\ ${ }^{4}$ Department of Thoracic Surgery and Respiratory Rehabilitation, Medical University \\ of Lodz, Lodz, Poland
}

Submitted: 20 February 2018

Accepted: 20 September 2018

Arch Med Sci Civil Dis 2018; 3: e180-e183

DOI: https://doi.org/10.5114/amscd.2018.81130

Copyright (c) 2018 Termedia \& Banach

Formation of an oropharyngeal or odontogenic abscess can spread the infection to the neck tissues and further via the cervical fascia planes to the mediastinum, leading to the development of cervical necrotizing fasciitis (CNF) and descending necrotizing mediastinitis (DNM) [1-7]. Clinically there are two forms of CNF: suppurative and gaseous [3, 4]. Criteria for the diagnosis of DNM were defined by Estrera et al. Endo et al. classified the degree of DNM diffusion based on CT findings [1, 3, 6-10]. The DNM is usually a polymicrobial process. Treatment options for CNF and DNM include antibiotic therapy, surgical drainage of the neck and mediastinum, airway management and debridement of infected tissues [2, 3, 5, 7, 9, $11,12]$. We describe a case of CNF with DNM in which dental treatment influenced the postoperative course.

A 34-year-old man presented to his local hospital with pain in the mandible and throat, facial swelling, and fever $\left(>38.5^{\circ} \mathrm{C}\right)$. On laryngoscopy, a laryngologist found hypopharyngeal and tonsillar edema and prescribed antibiotic (cefuroxime). After 7 days, the patient started to complain about cervical and chest pain, subcutaneous cervical emphysema, fever and mild odynophagia. He was sent to the Department of Infectious Diseases with suspicion of mononucleosis. A computed tomography (CT) scan revealed diffuse pharyngeal and cervical edema, gaseous spaces in the parapharyngeal region extending into the neck and upper mediastinum as well as focal pneumonia in lower lobes. The CNF and DNM diagnoses were established and the patient was referred to our hospital. The hemodynamically stable patient on admission presented with moderate right submandibular swelling. His neck was edematous, and crepitation and dullness on percussion in the right lower lung field were noted. White blood cells (WBC) count was $13.28 \times 10^{3} / \mu \mathrm{l}$, and C-reactive protein level was $237.50 \mathrm{mg} / \mathrm{l}$. The patient was taken to the operating room for incision and drainage of the neck. Drainage and irrigation of the superior mediastinum through right anterolateral thoracotomy were installed. The wound culture revealed Enterococcus faecalis (HLAR) so, after empiric amoxicillin/clavulanic acid, amikacin and metronidazole, vancomycin were administered. The culture did not reveal any anaerobic bacteria. Dressing changes and irrigation with saline, chlorhexidine and

\author{
Corresponding author: \\ Agnieszka Kozak-Rusinek MD \\ Department \\ of Maxillofacial Surgery \\ Medical University \\ of Lodz \\ 113 Żeromskiego St \\ 93-362 Lodz, Poland \\ Phone: +48 504226775 \\ E-mail: agnieszka.kozak@ \\ umed.lodz.pl
}


povidone-iodine through the chest tubes were performed. On day 5, a pharyngo-cutaneous fistula on the right side of the neck developed and was treated conservatively. The oral cavity was irrigated, and parenteral and enteral immunonutrition enriched with omega-3 fatty acids (Omegaven) and glutamine (Dipeptiven) was started. Additionally, peritonsillar swelling and erythema was observed in the patient's oropharynx. On day 8 , he was still febrile with WBC count $15.88 \times 10^{3} / \mu \mathrm{l}$ and C-reactive protein $216.80 \mathrm{mg} / \mathrm{l}$. The repeated CT scan showed inflammation in both lower lung lobes and stabilization of mediastinal infiltrations. Bronchoscopically taken culture from bronchoalveolar lavage revealed Serratia marcescens and Streptococcus pneumonia. In spite of the stabilization process in the mediastinum and neck, the patient was still febrile (WBC $15.68 \times 10^{3} / \mu$ l, CRP $268.12 \mathrm{mg} / \mathrm{l})$. Intra-oral examination by the maxillofacial surgeon revealed periodontitis in teeth 16 , $26,27,28,35,36,45$ together with subperiosteal abscesses and purulent foci which were formed in the superior and posterior areas. On day 15, all above-mentioned teeth were extracted. The patient's clinical status improved over the next few days. He was afebrile, WBC count was $10.25 \times 10^{3} / \mu \mathrm{l}$, CRP was $143.85 \mathrm{mg} / \mathrm{l}$. The pharyngo-cutaneous fistula healed on day 23 and the patient started oral feeding. The patient was discharged to his regional hospital for rehabilitation on postoperative day 28 and regularly returned for follow-up visits.

Descending necrotizing mediastinitis, first named and described by Pearse in 1938 [1], is the most life-threatening and still underestimated type of acute mediastinitis and includes cases with descending fast spreading polymicrobial infection from localized odontogenic or oropharyngeal abscesses. The DNM is regarded as a CNF complication. Early diagnosis of CNF is the key point in the treatment of this complicated disease. Our patient was first examined by the laryngologist and admitted to the Department of Infectious Diseases for 6 days with the suspicion of mononucleosis and signs of oropharyngeal infection. After 9 days of hospitalization, a CT scan revealed a neck abscess and finally mediastinitis, 16 days after the first symptoms were observed. Most authors state that 10 days is the average time from occurrence of the first symptoms to establishing the diagnosis $[2,11]$. We consider this as one of the life-saving key points: not to delay diagnosis of CNF. Risk of DNM in deep neck infection ranges from $1.5 \%$ to $3.6 \%$ [4]. Specific neck anatomy with retropharyngeal, pretracheal and perivascular spaces facilitates descent of the disease to the superior, anterior and posterior mediastinum under gravity and negative intrathoracic pressure. That is why DNM is a progressive, widely spreading disease with a poor outcome, high morbidity and mortality rate. Before 1990 , the mortality rate of DNM was 40-50\% [3, 8, 10-12]. Despite the use of antibiotics, progress in new technologies such as CT scanning and advanced surgical techniques such as video-assisted thoracic surgery (VATS) and radiologically guided drainage techniques, the mortality rate is still at a very high level, ranging from 19 to 47\% [3, 6, 8]. Uncontrolled diabetes mellitus, use of glucocorticosteroids, alcohol or drug addiction and other immunocompromising factors increasing the risk of septic shock can lead to a higher mortality rate ranging from $64 \%$ to $67 \%[3,8,12,13]$. Estrera et al. in 1983 established the criteria for diagnosis of DNM resulting from CNF: 1) clinical manifestations of severe infection, 2) demonstration of characteristic radiographic features such as mediastinal widening, mediastinal emphysema, mediastinal fluid collection with bubbles or an abscess with air fluid level, 3) documentation of necrotizing mediastinal infection at operation or post-mortem examination or both, and 4) establishment of the relationship of oropharyngeal or cervical infection with the development of the necrotizing mediastinal process $[1,2]$. Diagnosis of infection and abscess in the mandibular (especially the second and third molar region), peritonsillar or pharyngeal region can stop neck tissue necrosis leading to DNM. Erythema and initially disproportionally strong pain in the neck as well as facial swelling, odynophagia, dysphagia, airway and neck tissue edema are the main symptoms of CNF. Thorough examination of the oral cavity is essential and leads to diagnosis of the primary source of infection. Secondly, it is obvious that empirical antibiotic therapy must be initiated with broad-spectrum antibiotics and a culture for the sensitivity test must be taken. Findings usually revealed polymicrobial infection with anaerobes and aerobes. The culture must be taken repeatedly. Early intervention is crucial for effective treatment. Airway security, early surgical procedures such as abscess and cervical fascia incisions and drainages as well as medical optimization are mandatory and can decrease mortality [3]. Persistent levels of infection determinants such as C-reactive protein evaluation and white blood cell count can indicate some unresolved focal infections in the oropharynx. Reexamination of our patient revealed peridental infection, and the extraction of 6 teeth was performed. After this procedure, WBC and CRP levels decreased. Most surgeons worldwide make their decision on the type of surgical intervention using the classification of DNM according to Endo et al., which is based on CT scan findings. Endo et al. [10] divided DNM into 3 types: type I: the infection is localized to the upper mediastinum and above the tracheal bifurca- 
tion; type II A: the infection extends to the lower anterior mediastinum; type II B: the infection extends to the lower anterior and posterior mediastinum. The CT scan is the best radiological examination for DNM diagnosis and should be repeated during a further treatment to reveal persisting or newly formed fluid or pus. There are no controversies concerning surgical approach in type I. Transcervical mediastinal drainage, wide cervical incisions along the medial margin of sternocleidomastoid muscles and drainage are performed. It is well described that $60-70 \%$ of CNF cases are induced in the peritonsillar or peridental region but in most papers it is not mentioned what type of procedures are performed in the oral cavity [1]. In type II A, some authors prefer subxiphoid access to the anterior mediastinum and cervical drainage, unlike others who prefer sternotomy or clamshell thoracotomy $[2,3,7]$. Controversies exist on possible complications of these approaches such as sterna dehiscence or osteomyelitis, but Kocher et al. did not observe these complications in their group of 17 patients [9]. Most authors advise aggressive surgical treatment especially in type II $\mathrm{B}$, which is crucial and often life-saving [4, 13]. Posterolateral, anterolateral thoracotomy or the VATS approach are the most often performed procedures, similar to the above-mentioned anterior approaches in some centers. In several papers, the authors indicate that posterolateral thoracotomies and VATS need to be repeated unlike anterior procedures, but in severely ill patients with complications, VATS and anterolateral or posterolateral thoracotomy seem to be optimal [6]. Wide opening of the mediastinal pleura and the anterior and posterior mediastinum, as well as irrigation of pus and fluid and effective pleural and mediastinal drainage during these operations are performed. Some authors advise everyday irrigation of mediastinal and pleural spaces but some claim there is no scientific evidence to benefit from irrigations $[6,9,14]$. Apart from these controversies, there are unarguable points of treatment: early diagnosis, empirical broad-spectrum antibiotic therapy, aggressive early surgical interventions, and thorough surveillance which can improve the prognosis dramatically $[5,6]$. Some patients require tracheostomy, ICU stay and multidisciplinary treatment including the intensivist, laryngologist, as well as maxillofacial and thoracic surgeons [15]. Recently, some authors have reported on beneficial hyperbaric oxygen therapy $[4,11,12,14]$. Immunonutritional support (with omega-3 fatty acids and glutamine) seems to be beneficial as well, but it needs further investigations. There are some complications after surgical or improper treatment of DNM such as severe necrosis and damage to cervical tissues, tracheal stenosis, multi-organ failure, deep neck thrombosis, and pharyngo-cutaneous fistula. They need additional procedures during the patient's convalescence.

Early multidisciplinary aggressive treatment of oropharyngeal infection and cervical necrotizing fasciitis are life-saving procedures. Thorough surveillance with repeated CT scan, white blood cell count, C-reactive protein level, bacteriological culture monitoring and surgical procedures in descending necrotizing mediastinitis facilitate treatment and avoid complications.

\section{Acknowledgments}

Funding: grant no. 502-03/5-061-02/502-54-176.

\section{Conflict of interest}

The authors declare no conflict of interest.

\section{References}

1. Novakov IP, Safev GP, Peicheva SE. Descending necrotizing mediastinitis of odontogenic origin - personal experience and literature review. Folia Med (Plovdiv) 2010; 52: $13-20$.

2. Singhal P, Kejriwal N, Lin Z, Tsutsui R, Ullal R. Optimal surgical management of descending necrotising mediastinitis: our experience and review of literature. Heart Lung Circ 2008; 17: 124-8.

3. Sokouti M, Nezafati S. Descending necrotizing mediastinitis of oropharyngeal infections. J Dent Res Dent Clin Dent Prospects 2009; 3: 82-5.

4. Sarna T, Sengupta T, Miloro M, Kolokythas A. Cervical necrotizing fasciitis with descending mediastinitis: literature review and case report. J Oral Maxillofac Surg 2012; 70: 1342-50.

5. Diamantis S, Giannakopoulos H, Chou J, Foote J. Descending necrotizing mediastinitis as a complication of odontogenic infection. Int J Surg Case Rep 2011; 2: 65-7.

6. Chen KC, Chen JS, Kuo SW, et al. Descending necrotizing mediastinitis: a 10-year surgical experience in a single institution. J Thorac Cardiovasc Surg 2008; 136: 191-8.

7. Foroulis CN, Sileli MN. Descending necrotizing mediastinitis: review of the literature and controversies in management. Open Surg J 2011; 511: 12-8.

8. Cho JS, Kim YD, Lee SK, Jeong YJ. Treatment of mediastinitis using video-assisted thoracoscopic surgery. Eur J Cardiothorac Surg 2008; 34: 520-4.

9. Kocher GJ, Hoksch B, Caversaccio M, Wiegand J, Schmid RA. Diffuse descending necrotizing mediastinitis: surgical therapy and outcome in a single-centre series. Eur J Cranio Thorac Surg 2012; 42: 66-72.

10. Endo S, Murayama F, Hasegawa T, et al. Guideline of surgical management based on diffusion of descending necrotizing mediastinitis. Jpn J Thorac Cardiovasc Surg1999; 47: 14-9.

11. Karkas A, Chahine K, Schmerber S, Brichon PY, Righini CA. Optimal treatment of cervical necrotizing fasciitis associated with descending necrotizing mediastinitis. Br J Surg 2010; 97: 609-15.

12. Gonlugur U, Guclu O, Karatag O, Mirici A, Derekoy S. Cervical necrotizing fasciitis associated with descending necrotizing mediastinitis. Multidiscip Respir Med 2011; 6: 387-9. 
13. Petipas F, Blancal JP, Mateo J, et al. Factors associated with the mediastinal spread of cervical necrotizing fasciitis. Ann Thorac Surg 2012; 93: 234-8.

14. Sander A, Börgermann J, Kösling S, Silber RE, Bloching MB. Descending necrotising mediastinitis: early detection and radical surgery are crucial. J Oral Maxillofac Surg 2007; 65: 794-800.

15. Muhammad JK, Almadani H, Al Hashemi BA, Liaqat M. The value of early intervention and a multidisciplinary approach in the management of necrotizing fasciitis of the neck and anterior mediastinum of odontogenic origin. J Oral Maxillofac Surg 2015; 73: 918-27. 